\title{
LOCALLY PERIODIC HOMOGENIZATION OF REFLECTED DIFFUSION
}

\author{
ABOUBAKARY DIAKHABY AND YOUSSEF OUKNINE
}

Received 27 July 2005; Accepted 15 February 2006

We study the homogenization of reflected SDEs with locally periodic coefficients and highly oscillating drift. Our method is entirely probabilistic, and builds upon earlier works of Tanaka, Benchérif-Madani and Pardoux, and Bensoussan et al. We extend, to Tanaka's theorem locally periodic case.

Copyright (c) 2006 A. Diakhaby and Y. Ouknine. This is an open access article distributed under the Creative Commons Attribution License, which permits unrestricted use, distribution, and reproduction in any medium, provided the original work is properly cited.

\section{Introduction}

Let $L^{\varepsilon}$ be a uniformly elliptic second-order partial differential operator of the form (2.11) indexed by a parameter $\varepsilon>0$. The homogenization problem for an elliptic equation consists in computing the limit as $\varepsilon \downarrow 0$, of the solution $u_{\varepsilon}(x)$ of $L^{\varepsilon} u_{\varepsilon}=f$ in a domain $D$ of $\mathbb{R}^{d}$ subject to an appropriate boundary condition under the assumption that the coefficients $a^{i j}(x), b^{i}(x)$, and $c^{i}(x)$ are periodic, almost periodic, or more generally, stationary random fields. In a probabilistic approach the problem becomes the following. What is the limit of the laws of the diffusion processes $X_{t}^{\varepsilon}$ with generator $L^{\varepsilon}$ as $\varepsilon \rightarrow 0$ ? This kind of problem has been studied for diffusion processes in the whole of $\mathbb{R}^{d}$ by Freĭdlin $[5,6]$ and Bensoussan et al. [3], and in the case of the presence of boundary conditions by Tanaka [13]; and in this paper, we will consider the case of locally periodic coefficients and generalize [13, Theorem 2.2]. This result of Tanaka is used by Ouknine and Pardoux [9], the authors have combined the probabilistic approach of Pardoux [10] with backward stochastic differential equations, in order to derive homogenization results for semilinear parabolic PDEs with periodic highly oscillating drift and nonlinear term and nonlinear Neumann boundary conditions. We note that Benchérif-Madani and Pardoux $[1,2]$ deal in the locally periodic case with the same problem with a Cauchy boundary condition.

The paper is organized as follows. In Section 2, we give our assumptions, notation, and the problem formulation. In Section 3 we deal with the main result and its proof. 
2 Locally periodic homogenization of reflected diffusion

\section{Reflected diffusion with rapidly oscillating and locally periodic coefficients}

Let $D=\left\{\left(x^{1}, \ldots, x^{d}\right) \in \mathbb{R}^{d}, x^{1}>0\right\}$, the functions $\sigma: \mathbb{R}^{d} \otimes \mathbb{R}^{d} \rightarrow \mathbb{R}^{d} \otimes \mathbb{R}^{d}, b: \mathbb{R}^{d} \otimes \mathbb{R}^{d} \rightarrow$ $\mathbb{R}^{d}, c: \mathbb{R}^{d} \otimes \mathbb{R}^{d} \rightarrow \mathbb{R}^{d}$, and $\gamma: \partial D \otimes \partial D\left(\cong \mathbb{R}^{d-1} \otimes \mathbb{R}^{d-1}\right) \rightarrow \mathbb{R}^{d}$ are locally periodic (i.e., periodic with respect to the second variable; of period 1 in each direction in $D$ ), and $\gamma^{1}(x, y)=1$. We note $a=\sigma(x, y)^{t} \sigma(x, y) / 2$ and we define some family of operators indexed by $x$ and acting on $y$. By convention $\partial_{i}$ means $\partial_{y_{i}}$ :

$$
\begin{gathered}
L_{x}^{\epsilon}=\sum_{i, j=1}^{d} a^{i j}\left(x, \frac{y}{\varepsilon}\right) \partial_{i} \partial_{j}+\frac{1}{\varepsilon} \sum_{i=1}^{d} b^{i}\left(x, \frac{y}{\varepsilon}\right) \partial_{i}+\sum_{i=1}^{d} c^{i}\left(x, \frac{y}{\varepsilon}\right) \partial_{i}, \\
L_{x, y}=\sum_{i, j=1}^{d} a^{i j}(x, y) \partial_{i} \partial_{j}+\sum_{i=1}^{d} b^{i}(x, y) \partial_{i}, \\
\Gamma_{x}^{\epsilon}=\sum_{i=1}^{d} \gamma^{i}\left(x, \frac{y}{\varepsilon}\right) \partial_{i}, \\
\Gamma_{x, y}^{\varepsilon}=\sum_{i=1}^{d} \gamma^{i}(x, y) \partial_{i} .
\end{gathered}
$$

2.1. Assumptions on the coefficients. Our standing assumptions are the following.

(H.1) Global Lipschitz condition: there exists a constant $c$ such that for any $\zeta=a, b, c$, and $\gamma$,

$$
\left\|\zeta(x, y)-\zeta\left(x^{\prime}, y^{\prime}\right)\right\| \leq c\left(\left\|x-x^{\prime}\right\|+\left\|y-y^{\prime}\right\|\right) \quad \forall x, x^{\prime} \in \mathbb{R}^{d} ; y, y^{\prime} \in \mathbf{T}^{d} .
$$

(H.2) The partial derivatives $\partial_{x} \zeta(x, y)$ as well as the mixed derivatives $\partial_{x y}^{2} \zeta(x, y)$ exist and are continuous, $\zeta=a, b, c$, and $\gamma, x \in \mathbb{R}^{d}, y \in \mathbf{T}^{d}$.

(H.3) The coefficients are bounded, that is, there exists a constant $c$ such that for any $\zeta=a, b, c$, and $\gamma$,

$$
\|\zeta(x, y)\| \leq c, \quad x \in \mathbb{R}^{d}, y \in \mathbf{T}^{d} .
$$

The system

$$
\begin{gathered}
L_{x}^{\epsilon} \quad \text { inside } D, \\
\Gamma_{x}^{\epsilon} u=0 \quad \text { on } \partial D
\end{gathered}
$$

determines a unique diffusion process on $\bar{D}$, which is called $\left(L_{x}^{\epsilon}, \Gamma_{x}^{\epsilon}\right)$-diffusion.

By requirement there exist a $L_{x, y}$-diffusion on $\mathbb{R}^{d}$ with generator $L_{x, y}$ and by $Y$-periodicity assumption on the coefficients this process induces diffusion process $U^{x}$ on the $d$-dimensional torus $\mathbf{T}^{d}$, moreover this diffusion process is ergodic. We denote by $m(x, \cdot)$ its unique invariant measure. In order for the process with generator $L_{x}^{\epsilon}$ to have a limit in law as $\varepsilon \rightarrow 0$, we need the following condition to be in force. 
(H.4) Centering condition: for all $x$,

$$
\int_{\mathbf{T}^{d}} b(x, u) m(x, d u)=0 .
$$

2.2. Notations. We use the following notation for any functions $\zeta(x, y)$ or $\xi(x)$ :

$$
\begin{gathered}
\zeta\left(X_{s}^{\epsilon}, \frac{X_{t}^{\epsilon}}{\epsilon}\right) \equiv \zeta(s, t), \\
\Delta_{s, r} \xi(\cdot) \equiv \xi(r)-\xi(s), \\
\partial_{i} \zeta(x, y) \equiv \partial_{y_{i}} \zeta(x, y) .
\end{gathered}
$$

First we notice that under (H.4), there exists a unique periodic solution $\hat{b}^{k}$ of $L \hat{b}^{k}=-b^{k}$ for each $k=1, \ldots, d$, with zero integral against the measure $m(x, \cdot)$. That solution is given by $\hat{b}^{k}(x, u)=\int_{0}^{\infty} \mathbb{E}_{u}\left\{b^{k}\left(x, U_{t}^{x}\right)\right\} d t$ where under $\mathbb{P}_{u}, U^{x}$ starts from $u$.

We set

$$
\begin{gathered}
\hat{b}=\left[\begin{array}{c}
\hat{b}^{1} \\
\vdots \\
\hat{b}^{d}
\end{array}\right], \quad \nabla_{y} \hat{b}=\left[\begin{array}{c}
\partial_{1} \hat{b}^{1} \cdots \partial_{d} \hat{b}^{1} \\
\vdots \ldots \\
\partial_{1} \hat{b}^{d} \cdots \partial_{d} \hat{b}^{d}
\end{array}\right], \\
a_{0}(x)=\int_{T^{d}}\left(I+\nabla_{y} \hat{b}\right) a^{t}\left(I+\nabla_{y} \hat{b}\right)(x, u) m(x, d u), \\
c_{0}(x)=\int_{T^{d}}\left(I+\nabla_{y} \hat{b}\right) c(x, u) m(x, d u), \\
L^{0}(x)=\sum_{i, j=1}^{d} a_{0}^{i j}(x) \partial_{i} \partial_{j}+\sum_{i=1}^{d} c_{0}^{i}(x) \partial_{i} .
\end{gathered}
$$

We write $u=H^{x} \varphi$ for the solution $u$ of

$$
\begin{aligned}
& L_{x} u=0 \text { in } D \\
& u=\varphi \text { on } \partial D .
\end{aligned}
$$

Then $H^{x}$ sends functions defined on $\partial D$ to functions defined on $\bar{D}$, while $\Gamma^{x} H$ sends functions on $\partial D$ to functions on $\partial D$, where $\Gamma^{x}=\sum_{i=1}^{d} \gamma^{i}(x, y) \partial_{i}$. There exist a unique Markov process on $\partial D$ with generator $\Gamma^{x} H^{x}$. By the periodicity assumption this Markov process induces a Markov process on the torus $T^{d-1}$; let $\tilde{m}(x, \cdot)$ be the invariant measure of the induced Markov process. We set

$$
\begin{gathered}
\gamma_{0}(x)=\int_{\mathrm{T}^{d-1}}\left(I+\nabla_{y} \hat{b}\right) \gamma(x, u) \tilde{m}(x, d u), \\
\Gamma^{0}(x)=\sum_{i=1}^{d} \gamma_{0}^{i}(x) \partial_{i} .
\end{gathered}
$$


4 Locally periodic homogenization of reflected diffusion

Given a $d$-dimensional Brownian motion $\left\{B_{t} ; t \geq 0\right\}$ defined on a probability space $(\Omega, \mathscr{F}$, $\mathbb{P}),\left(X^{\varepsilon}, \phi^{\varepsilon}\right)$ is the unique solution with values in $D \times \mathbb{R}_{+}$of the following reflected SDE:

$$
\begin{gathered}
d X_{t}^{\varepsilon}=\sigma\left(X_{t}^{\varepsilon}, \frac{X_{t}^{\varepsilon}}{\varepsilon}\right) d B_{t}+\frac{1}{\varepsilon} b\left(X_{t}^{\varepsilon}, \frac{X_{t}^{\varepsilon}}{\varepsilon}\right) d t+c\left(X_{t}^{\varepsilon}, \frac{X_{t}^{\varepsilon}}{\varepsilon}\right) d t+\gamma\left(X_{t}^{\varepsilon}, \frac{X_{t}^{\varepsilon}}{\varepsilon}\right) d \phi_{t}^{\varepsilon}, \quad t \geq 0, \\
X_{t}^{\varepsilon, 1} \geq 0, \quad \phi^{\varepsilon} \text { is continuous and increasing, } \quad \int_{0}^{t} X_{s}^{1, \varepsilon} d \phi_{s}^{\varepsilon}=0, \quad t \geq 0,
\end{gathered}
$$

$$
X_{0}^{\varepsilon}=x
$$

where $X^{\varepsilon, 1}$ denotes the first component of the process $X^{\varepsilon}$. We recall that $D=\mathbb{R}_{+}^{d}$, so that $X^{\varepsilon}$ lives in $D$, that is, $X^{\varepsilon, 1}$ remains nonnegative, and $\phi^{\varepsilon}$ increases when and only when $X^{\varepsilon, 1}$ is zero, just to keep it nonnegative.

Let

$$
\begin{gathered}
L^{\epsilon}=\sum_{i, j=1}^{d} a^{i j}\left(x, \frac{x}{\varepsilon}\right) \partial_{x_{i}} \partial_{x_{j}}+\frac{1}{\varepsilon} \sum_{i=1}^{d} b^{i}\left(x, \frac{x}{\varepsilon}\right) \partial_{x_{i}}+\sum_{i=1}^{d} c^{i}\left(x, \frac{x}{\varepsilon}\right) \partial_{x_{i}}, \\
\Gamma^{\epsilon}=\sum_{i=1}^{d} \gamma^{i}\left(x, \frac{x}{\varepsilon}\right) \partial_{x_{i}}
\end{gathered}
$$

be the operators acting on $x$, so the diffusion process $X_{t}^{\varepsilon}$ is an $\left(L^{\epsilon}, \Gamma^{\epsilon}\right)$-diffusion.

We define

$$
\begin{gathered}
C_{0}(x, y)=\left[\partial_{x} \hat{b} b+\left(I+\partial_{y} \hat{b}\right) c+\frac{1}{2} \operatorname{Tr} \partial_{x y}^{2} \hat{b} \sigma \sigma^{*}\right](x, y), \\
S_{0}(x, y)=\left[\left(I+\partial_{y} \hat{b}\right) \sigma\right](x, y), \quad A_{0}(x, y)=S_{0} S_{0}^{*}(x, y), \\
\bar{C}_{0}(x)=\int_{\mathrm{T}^{d}} C_{0}(x, u) m(x, d u), \quad \bar{A}_{0}(x)=\int_{\mathrm{T}^{d}} A_{0}(x, u) m(x, d u), \\
\gamma_{0}(x)=\int_{\mathrm{T}^{d-1}}\left[\left(I+\partial_{y} \hat{b}\right) \gamma\right](x, y) \tilde{m}(x, d y), \\
L^{0}=\sum_{i, j=1}^{d} \bar{A}_{0}^{i j}(x) \partial_{x_{i}} \partial_{x_{j}}+\sum_{i=1}^{d} \bar{C}_{0}^{i}(x) \partial_{x_{i}}, \quad \Gamma^{0}=\sum_{i=1}^{d} \gamma_{0}^{i}(x) \partial_{x_{i}}, \\
d X_{t}=\bar{A}_{0}^{1 / 2}\left(X_{t}\right) d B_{t}+\bar{C}_{0}\left(X_{t}\right) d t+\gamma_{0}\left(X_{t}\right) d \phi_{t}, \quad t \geq 0,
\end{gathered}
$$$$
X_{t}^{1} \geq 0, \quad \phi \text { is continuous and increasing, } \quad \int_{0}^{t} X_{s}^{1} d \phi_{s}=0, \quad t \geq 0,
$$

$$
X_{0}=x
$$

The operators $L^{0}$ and $\Gamma^{0}$ are acting on $x$. 


\section{Main result}

We can now state our main results, which are a generalization of Tanaka [13, Theorem 2.2].

Theorem 3.1. Under the assumptions (H.1), (H.2), (H.3), and (H.4), the $\left(L^{\varepsilon}, \Gamma^{\varepsilon}\right)$-diffusion process $X^{\varepsilon}$ converges in law to an $\left(L^{0}, \Gamma^{0}\right)$-diffusion $X$ as $\varepsilon \downarrow 0$. Moreover,

$$
\left(X^{\varepsilon}, M^{X^{\varepsilon}}, \phi^{\varepsilon}\right) \Longrightarrow\left(X, M^{X}, \phi\right),
$$

where $M^{X}$ (resp., $M^{X^{\varepsilon}}$ ) is the martingale part of $X$ (resp., $\left.X^{\varepsilon}\right)$, and $\phi$ (resp., $\left.\phi^{\varepsilon}\right)$ is the local time of $X^{1}$ (resp., $\left.X^{\varepsilon, 1}\right)$ at 0 .

Remark 3.2 (Skorohod equation). We have $X_{s}^{\varepsilon, 1}=U_{s}^{\epsilon, 1}+\phi_{s}^{\varepsilon}$, where

$$
U_{t}^{\epsilon}=x+\int_{0}^{t}\left(\sigma\left(X_{s}^{\varepsilon}, \frac{X_{s}^{\varepsilon}}{\varepsilon}\right) d B_{s}+\frac{1}{\varepsilon} b\left(X_{s}^{\varepsilon}, \frac{X_{s}^{\varepsilon}}{\varepsilon}\right) d s+c\left(X_{s}^{\varepsilon}, \frac{X_{s}^{\varepsilon}}{\varepsilon}\right) d s\right),
$$

so by [13, Proposition 3.2],

$$
\phi_{t}^{\varepsilon}-\phi_{s}^{\varepsilon} \leq \max _{s \leq t_{1} \leq t_{2} \leq t}\left|U_{t_{1}}^{\epsilon, 1}-U_{t_{2}}^{\epsilon, 1}\right| \leq \sup _{s \leq t_{1} \leq t_{2} \leq t}\left\|U_{t_{2}}^{\epsilon}-U_{t_{1}}^{\epsilon}\right\|, \quad 0 \leq s \leq t,
$$

and using the boundedness of the coefficients, with probability one, we have

$$
\phi_{t}^{\varepsilon}-\phi_{s}^{\varepsilon} \leq c\left((t-s)+(t-s)^{1 / 2}+\epsilon^{-1}(t-s)\right), \quad 0 \leq s \leq t,
$$

and for $t-s \leq \epsilon^{2}$,

$$
\phi_{t}^{\varepsilon}-\phi_{s}^{\varepsilon} \leq c \epsilon(2+\epsilon) \quad \forall t, s, 0 \leq s \leq t \leq s+\epsilon^{2} \leq T .
$$

Let $U$. be the unique diffusion in $\mathbb{R}^{d}$, solution in law to the stochastic differential equation for $0 \leq t \leq T$,

$$
U_{t}=\int_{0}^{t} \bar{C}_{0}\left(U_{s}\right) d s+\int_{0}^{t} \bar{A}_{0}^{1 / 2}\left(U_{s}\right) d B_{s}
$$

Then we have from [1], that $U^{\epsilon}$ converge in law sense to $U$ (i.e., $U^{\epsilon} \Rightarrow U$ ). We have, by the Skorohod equation, that $\left(X^{\varepsilon, 1}, \phi^{\varepsilon}\right)$ is associated to $U^{\varepsilon, 1}$ :

$$
X^{\varepsilon, 1}=U^{\varepsilon, 1}+\phi^{\varepsilon} \quad \forall \epsilon>0 .
$$

According to the above result, the above remark, and Słomiński [11, Corollary A.3], we have the following.

Lemma 3.3. $\left(X^{\varepsilon, 1}, U^{\varepsilon, 1}, \phi^{\varepsilon}\right) \Rightarrow\left(X^{1}, U^{1}, \phi\right)$ where $\left(X^{1}, \phi\right)$ is associated to $U^{1}$.

By [1, Lemmas 21 and 22] and the above remark, we have also the following.

Lemma 3.4. For any $p>0$, there exists a constant $c_{p}$ such that for all $\varepsilon>0$,

$$
\mathbb{E}\left(\phi_{T}^{\varepsilon}\right)^{p}<c_{p} .
$$


6 Locally periodic homogenization of reflected diffusion

Proof. We have from the remark that $X_{s}^{\varepsilon, 1}=U_{s}^{\epsilon, 1}+\phi_{s}^{\varepsilon}$ and since $U_{t}^{\epsilon}=\overline{U_{t}^{\epsilon}}+\mathbb{R}_{t}^{\varepsilon}$ by $[1$, Lemmas 21 and 22], for any $0 \leq t \leq T, \exists c$ such that $\mathbb{E}\left\|U_{t}^{\epsilon}\right\|^{p}<c$ and $\mathbb{E}\left\|\mathbb{R}_{t}^{\epsilon}\right\|^{p}<c$. From (3.3), we have $\mathbb{E}\left(\phi_{T}^{\varepsilon}\right)^{p} \leq \mathbb{E}\left(\max _{0 \leq t_{1} \leq t_{2} \leq t}\left|U_{t_{1}}^{\epsilon, 1}-U_{t_{2}}^{\epsilon, 1}\right|\right)^{p} \leq \mathbb{E}\left(\sup _{0 \leq t_{1} \leq t_{2} \leq t}\left|U_{t_{2}}^{\epsilon}-U_{t_{1}}^{\epsilon}\right|\right)^{p}<\infty$.

From [1, Lemma 1] it is easy to reach the following result.

Lemma 3.5. Let $h(x, y)$ be a continuous bounded function on $\mathbb{R}^{d} \times \mathbf{T}^{d}$ such that for all $x \in \mathbb{R}^{d}, \int_{\mathrm{T}^{d}} h(x, u) m(x, d u)=0$. Then

$$
\begin{aligned}
\epsilon^{-1} \int_{s}^{t} h(r, r) d r= & \epsilon^{-1} \int_{s}^{t}\left(\Delta_{s, r} h(\cdot, r)+\Delta_{s, r} L \cdot, r \hat{h}(s, r)\right) d r \\
& +\int_{s}^{t} \partial_{y} \hat{h}(s, r)\left[c(r, r) d r+\gamma(r, r) d \phi_{r}^{\epsilon}\right] \\
& +\int_{s}^{t} \partial_{y} \hat{h}(s, r) \sigma(r, r) d B_{r}+\epsilon \Delta_{t, s} \hat{h}(s, \cdot) .
\end{aligned}
$$

Proof. Use the Itô-Krylov formula to compute $\epsilon \Delta_{s, t} \hat{h}(s, \cdot)$, where $\hat{h}$ is the solution of the Poisson equation, and the fact that $L_{s, r} \hat{h}(s, r)+h(s, r)=0$.

Let us take a fine enough equidistant subdivision, ultimately depending on $\epsilon$, of the interval $[0, T]$ by means of the points $t_{i}, i=0, \ldots,[T / \Delta t]=\mathbb{N}$, where $t_{0}=0, \Delta t_{i}=t_{i+1}-t_{i}$. We denote by $t_{*}$ the largest $t_{i}$ below $t$, by $t^{*}$ the least $t_{i}$ above $t$, and by $\mathbb{N}_{t}$ the integer $[t / \Delta t]$ for $t \leq T$. Applying the preceding lemma to $b(x, y)$ on each $\Delta t_{i}$ we can derive a representation of $X_{t}^{\varepsilon}$ in which the singularity is removed by introducing a multiplicative small corrector term.

Let us first define, for $0 \leq s \leq T$,

$$
\begin{aligned}
& F^{0, \epsilon}\left(s_{*}, s\right)=\left(I+\partial_{y} \hat{b}\left(s_{*}, s\right)\right) c(s, s), \\
& G^{0, \epsilon}\left(s_{*}, s\right)=\left(I+\partial_{y} \hat{b}\left(s_{*}, s\right)\right) \sigma(s, s), \\
& \gamma^{0, \epsilon}\left(s_{*}, s\right)=\left(I+\partial_{y} \hat{b}\left(s_{*}, s\right)\right) \gamma(s, s), \\
& \mathbb{R}^{0, \epsilon}\left(s_{*}, s\right)=\Delta_{s_{*}, s} b(\cdot, s)+\Delta_{s_{*}, s} L,{ }_{, s} \hat{b}\left(s_{*}, s\right),
\end{aligned}
$$

and state the following as in [1].

Corollary 3.6. With the notation above, for $0 \leq t \leq T$,

$$
\begin{aligned}
X_{t_{*}}^{\varepsilon}= & x+\int_{0}^{t_{*}} F^{0, \epsilon}\left(s_{*}, s\right) d s+\int_{0}^{t_{*}} G^{0, \epsilon}\left(s_{*}, s\right) d B_{s}+\varepsilon^{-1} \int_{0}^{t_{*}} \mathbb{R}^{0, \epsilon}\left(s_{*}, s\right) d s \\
& +\varepsilon \sum_{i=0}^{\mathbb{N}_{t}-1} \Delta_{t_{i+1}, t_{i}} \hat{b}\left(t_{i}, \cdot\right)+\int_{0}^{t_{*}} \gamma^{0, \epsilon}\left(s_{*}, s\right) d \phi_{s}^{\epsilon} .
\end{aligned}
$$

Proof. Write down $X_{t_{*}}^{\varepsilon}$ and use Lemma 3.5 to change $\epsilon^{-1} \int_{0}^{t_{*}} b(s, s) d s$ by $\sum_{i=0}^{\mathbb{N}_{t}-1} \epsilon^{-1} \int_{t_{i}}^{t_{i+1}} b(s$, s)ds. 
We need the following.

LEMMA 3.7 [1, Lemma 2]. Under the conditions above, there exists a constant $c>0$ such that for all $x \in \mathbb{R}^{d}$ and $y$ in $\mathbf{T}^{d}$,

$$
\|\hat{b}(x, y)\|+\left\|\partial_{x} \hat{b}(x, y)\right\|+\left\|\partial_{y} \hat{b}(x, y)\right\|+\left\|\partial_{y}^{2} \hat{b}(x, y)\right\|+\left\|\partial_{x y}^{2} \hat{b}(x, y)\right\| \leq c
$$

and these derivatives are continuous.

Now we can give a result about tightness.

LEMma 3.8. There exists a constant $c$ such that for all $\epsilon>0$ and $0 \leq s<t \leq T$,

$$
\mathbb{E}\left(\sup _{s \leq v \leq t}\left\|X_{v}^{\varepsilon}-X_{s}^{\varepsilon}\right\|^{4}\right) \leq c\left[(t-s)^{2}+\epsilon^{4}+\mathbb{E}\left(\phi_{t}^{\epsilon}-\phi_{r}^{\epsilon}\right)^{4}\right]
$$

Proof. Let $t_{i}$ be as in Corollary 3.6 and let $0 \leq s<v \leq t \leq T$, we can write

$$
\left\|X_{v}^{\varepsilon}-X_{s}^{\varepsilon}\right\| \leq\left\|X_{v}^{\varepsilon}-X_{v_{*}}^{\varepsilon}\right\|+\left\|X_{v_{*}}^{\varepsilon}-X_{s_{*}}^{\varepsilon}\right\|+\left\|X_{s}^{\varepsilon}-X_{s_{*}}^{\varepsilon}\right\| .
$$

By Lemmas 3.5 and 3.7, for $0 \leq r_{*} \leq v \leq r \leq T$,

$$
\left\|X_{v}^{\varepsilon}-X_{v_{*}}^{\varepsilon}\right\| \leq c\left(\begin{array}{c}
\epsilon^{-1} \int_{r_{*}}^{v}\left\|X_{u}^{\varepsilon}-X_{r_{*}}^{\varepsilon}\right\| d u+\left(v-r_{*}\right) \\
+\left\|\int_{r_{*}}^{v} G^{0, \epsilon}\left(r_{*}, u\right) d B_{u}\right\|+\epsilon+\left(\phi_{v}^{\epsilon}-\phi_{r_{*}}^{\epsilon}\right)
\end{array}\right) .
$$

Therefore by Hölder and convexity,

$$
\left\|X_{v}^{\varepsilon}-X_{v_{*}}^{\varepsilon}\right\|^{4} \leq c\left(\begin{array}{c}
\epsilon^{-4}\left(v-r_{*}\right)^{3} \int_{r_{*}}^{v}\left\|X_{u}^{\varepsilon}-X_{r_{*}}^{\varepsilon}\right\|^{4} d u+\left(v-r_{*}\right)^{4} \\
+\left\|\int_{r_{*}}^{v} G^{0, \epsilon}\left(r_{*}, u\right) d B_{u}\right\|^{4}+\epsilon^{4}+\left(\phi_{v}^{\epsilon}-\phi_{r_{*}}^{\epsilon}\right)^{4}
\end{array}\right) .
$$

Hence

$$
\mathbb{E}\left(\sup _{r_{*} \leq v \leq r}\left\|X_{v}^{\varepsilon}-X_{r_{*}}^{\varepsilon}\right\|^{4}\right) \leq c\left(\begin{array}{l}
\epsilon^{-4}\left(r-r_{*}\right)^{3} \int_{r_{*}}^{r} \mathbb{E}\left(\sup _{r_{*} \leq v \leq u}\left\|X_{v}^{\varepsilon}-X_{r_{*}}^{\varepsilon}\right\|^{4}\right) d u \\
+\left(r-r_{*}\right)^{4}+\left(r-r_{*}\right)^{2}+\epsilon^{4}+\mathbb{E}\left(\phi_{r}^{\epsilon}-\phi_{r_{*}}^{\epsilon}\right)^{4}
\end{array}\right) .
$$


8 Locally periodic homogenization of reflected diffusion

By the Gronwall-Bellman lemma,

$$
\mathbb{E}\left(\sup _{r_{*} \leq v \leq r}\left\|X_{v}^{\varepsilon}-X_{r_{*}}^{\varepsilon}\right\|^{4}\right) \leq c\left(\begin{array}{c}
\left(r-r_{*}\right)^{4}+\left(r-r_{*}\right)^{2}+\epsilon^{4} \\
+\mathbb{E}\left(\phi_{r}^{\epsilon}-\phi_{r_{*}}^{\epsilon}\right)^{4}
\end{array}\right) e^{c^{\prime} \epsilon^{-4}\left(r-r_{*}\right)^{4}}
$$

We now choose $\Delta t_{i}=\epsilon^{2}$, by this and (3.5)

$$
\mathbb{E}\left(\sup _{r_{*} \leq v \leq r}\left\|X_{v}^{\varepsilon}-X_{r_{*}}^{\varepsilon}\right\|^{4}\right) \leq c\left[\epsilon^{4}+\mathbb{E}\left(\phi_{r}^{\epsilon}-\phi_{r_{*}}^{\epsilon}\right)^{4}\right] \leq c^{\prime} \epsilon^{4}
$$

Since, from Lemma 3.7, the function $\hat{b}(\cdot, y)$ is Lipschitz on $\mathbb{R}^{d}$ uniformly in $y \in \mathbf{T}^{d}$, we have by convexity for $s \leq v \leq t$,

$$
\mathbb{E}\left[\sup _{s \leq v \leq t}\left\|\sum_{i=\mathbb{N}_{s}+1}^{\mathbb{N}_{t}-1} \Delta_{t_{i+1}, t_{i}} \hat{b}\left(\cdot, t_{i}\right)\right\|^{4}\right] \leq c \mathbb{E}\left(\sum_{i=\mathbb{N}_{s}+1}^{\mathbb{N}_{t}-1}\left\|X_{t_{i}}^{\varepsilon}-X_{t_{i-1}}^{\varepsilon}\right\|\right)^{4} \leq c\left(\frac{t-s}{\Delta t_{i}}\right)^{4}\left[c^{\prime} \epsilon^{4}\right] .
$$

Hence

$$
\mathbb{E}\left[\sup _{s \leq v \leq t}\left\|\epsilon \sum_{i=\mathbb{N}_{s}+1}^{\mathbb{N}_{t}-1} \Delta_{t_{i+1}, t_{i}} \hat{b}\left(\cdot, t_{i}\right)\right\|^{4}\right] \leq c^{\prime \prime}(t-s)^{4}
$$

So by Corollary 3.6, we have

$$
\begin{aligned}
\mathbb{E}\left(\sup _{s \leq v \leq t}\left\|X_{v_{*}}^{\varepsilon}-X_{s_{*}}^{\varepsilon}\right\|^{4}\right) & \leq c\left[\epsilon^{4}+\mathbb{E}\left(\phi_{t}^{\epsilon}-\phi_{s}^{\epsilon}\right)^{4}+(t-s)^{2}+(t-s)^{4}\right] \\
& \leq c^{\prime}\left(\epsilon^{4}+(t-s)^{2}+\mathbb{E}\left(\phi_{t}^{\epsilon}-\phi_{s}^{\epsilon}\right)^{4}\right)
\end{aligned}
$$

which implies the result.

We can now state the following.

Theorem 3.9. Under the assumptions on the coefficients, the family of processes $\left\{X^{\varepsilon}\right.$, $0<\epsilon \leq 1\}$ is tight in $C[0, T]$.

Proof. By Billingsley [4, Theorem 8.3], it suffices to check that for any $\alpha$ and $\delta>0$, there exist $0<\epsilon_{0} \leq 1$ and $0<\theta \leq T$ such that

$$
\theta^{-1} \mathbb{P}\left(\sup _{s \leq \nu \leq s+\theta}\left\|X_{v}^{\varepsilon}-X_{s}^{\varepsilon}\right\|>\delta\right)<\alpha
$$

for all $s \leq T-\theta$ and $\epsilon \leq \epsilon_{0}$. We have by the Markov-Chebychev inequality

$$
\mathbb{P}\left(\sup _{s \leq v \leq s+\theta}\left\|X_{v}^{\varepsilon}-X_{s}^{\varepsilon}\right\|>\delta\right) \leq \frac{1}{\delta^{4}} \mathbb{E}\left(\sup _{s \leq v \leq s+\theta}\left\|X_{v}^{\varepsilon}-X_{s}^{\varepsilon}\right\|\right)^{4} \leq \frac{c}{\delta^{4}}\left[\theta^{2}+\epsilon^{4}+\mathbb{E}\left(\phi_{s+\theta}^{\epsilon}-\phi_{s}^{\epsilon}\right)^{4}\right] .
$$


By continuity of $\phi_{t}^{\epsilon}$, there exist $\theta_{0}$ such that $\mathbb{E}\left(\phi_{s+\theta}^{\epsilon}-\phi_{s}^{\epsilon}\right) \leq \epsilon$ (by (3.5), we have $\mathbb{E}\left(\phi_{t}^{\varepsilon}-\right.$ $\left.\left.\phi_{s}^{\varepsilon}\right) \leq c \epsilon, 0 \leq s \leq t \leq s+\epsilon^{2}\right)$ for all $0<\theta \leq \theta_{0} \leq T$. For any $\alpha$ and $\delta>0$, there exist $0<\epsilon_{0} \leq$ 1 and $0<\theta \leq \theta_{0} \leq T$ such that $c \theta\left[\theta^{2}+2 \epsilon^{4}\right]<\alpha \delta^{4}$ for $0<\epsilon<\epsilon_{0}$, this ends the proof.

We can recover our processes as a main term which converges in law, plus an asymptotically small term. By Corollary 3.6, we have

$$
\begin{aligned}
X_{t_{*}}^{\varepsilon}= & x+\int_{0}^{t_{*}}\left(I+\partial_{y} \hat{b}\left(s_{*}, s\right)\right) c(s, s) d s+\int_{0}^{t_{*}}\left(I+\partial_{y} \hat{b}\left(s_{*}, s\right)\right) \sigma(s, s) d B_{s} \\
& +\int_{0}^{t_{*}}\left(I+\partial_{y} \hat{b}\left(s_{*}, s\right)\right) \gamma(s, s) d \phi_{s}^{\epsilon}+\varepsilon \sum_{i=0}^{\mathbb{N}_{t}-1} \Delta_{t_{i+1}, t_{i}} \hat{b}\left(t_{i}, \cdot\right) \\
& +\sum_{i=0}^{\mathbb{N}_{t}-1} \varepsilon^{-1} \int_{\Delta t_{i}}\left(\Delta_{t_{i}, s} b(\cdot, s)+\Delta_{t_{i}, s} L \cdot, \hat{b}\left(t_{i}, s\right)\right) d s
\end{aligned}
$$

\section{Define}

$$
\begin{gathered}
F_{2}^{1, \epsilon}(s) \triangleq\left(I+\partial_{y} \hat{b}\left(s_{*}, s\right)\right) c(s, s), \\
G^{1, \epsilon}(s) \triangleq\left(I+\partial_{y} \hat{b}\left(s_{*}, s\right)\right) \sigma(s, s), \\
\gamma^{1, \epsilon}(s) \triangleq\left(I+\partial_{y} \hat{b}\left(s_{*}, s\right)\right) \gamma(s, s), \\
\mathbb{R}_{\mathbb{N}_{t}}^{1, \epsilon} \triangleq \sum_{i=0}^{\mathbb{N}_{t}-1} \varepsilon^{-1} \int_{\Delta t_{i}}\left(\Delta_{t_{i}, s} b(\cdot, s)+\Delta_{t_{i}, s} L \cdot, \hat{b}\left(t_{i}, s\right)\right) d s, \\
S_{\mathbb{N}_{t}}^{1, \epsilon} \sum_{i=0}^{\mathbb{N}_{t}-1} \Delta_{t_{i+1}, t_{i}} \hat{b}\left(t_{i}, \cdot\right)=\varepsilon \sum_{i=1}^{\mathbb{N}_{t}-1} \Delta_{t_{i-1}, t_{i}} \hat{b}\left(\cdot, t_{i}\right)+\epsilon\left(\hat{b}(0,0)-\hat{b}\left(t_{\mathbb{N}_{t}-1}, t_{\mathbb{N}_{t}}\right)\right) \\
\triangleq S_{\mathbb{N}_{t}}^{2, \epsilon}+\mathbb{R}_{\mathbb{N}_{t}}^{2, \epsilon} .
\end{gathered}
$$

So we have now

$$
\begin{gathered}
X_{t_{*}}^{\varepsilon}=x+\int_{0}^{t_{*}} F_{2}^{1, \epsilon}(s) d s+\int_{0}^{t_{*}} G^{1, \epsilon}(s) d B_{s}+\int_{0}^{t_{*}} \gamma^{1, \epsilon}(s) d \phi_{s}^{\epsilon}+S_{\mathbb{N}_{t}}^{1, \epsilon}+\mathbb{R}_{\mathbb{N}_{t}}^{1, \epsilon}, \\
S_{\mathbb{N}_{t}}^{1, \epsilon} \triangleq S_{\mathbb{N}_{t}}^{2, \epsilon}+\mathbb{R}_{\mathbb{N}_{t}}^{2, \epsilon} .
\end{gathered}
$$


10 Locally periodic homogenization of reflected diffusion

Using the same decomposition as in [1, pages $11-13$ of the preprint], we denote by $F_{1}^{2, \epsilon}(s)$ the process defined on $\left.] t_{i-1}, t_{i}\right]$ by $\partial_{x} \hat{b}\left(t_{i-1}, t_{i}\right) b(s, s)$.

We define

$$
\begin{aligned}
\mathbb{R}_{\mathbb{N}_{t}}^{3, \epsilon}= & \epsilon \sum_{i=1}^{\mathbb{N}_{t}-1} \int_{0}^{1}\left(\partial_{x} \hat{b}\left(X_{t_{i-1}}^{\varepsilon}+l \Delta_{t_{i-1}, t_{i}} X_{\cdot}^{\varepsilon}, \frac{X_{t_{i}}^{\varepsilon}}{\varepsilon}\right)-\partial_{x} \hat{b}\left(t_{i-1}, t_{i}\right)\right) \Delta_{t_{i-1}, t_{i}} X^{\varepsilon} d l \\
& +\epsilon \sum_{i=1}^{\mathbb{N}_{t}-1} \int_{t_{i-1}}^{t_{i}} \partial_{x} \hat{b}\left(t_{i-1}, t_{i}\right) c(s, s) d s+\epsilon \sum_{i=1}^{\mathbb{N}_{t}-1} \int_{t_{i-1}}^{t_{i}} \partial_{x} \hat{b}\left(t_{i-1}, t_{i}\right) \gamma(s, s) d \phi_{s}^{\varepsilon} \\
\triangleq & \mathbb{R}_{\mathbb{N}_{t}}^{3, \epsilon, 1}+\mathbb{R}_{\mathbb{N}_{t}}^{3, \epsilon, 2}+\mathbb{R}_{\mathbb{N}_{t}}^{3, \epsilon, 3}, \\
S_{\mathbb{N}_{t}}^{3, \epsilon}= & \epsilon \sum_{i=1}^{\mathbb{N}_{t}-1} \partial_{x} \hat{b}\left(t_{i-1}, t_{i}\right) \int_{t_{i-1}}^{t_{i}} \sigma(s, s) d B_{s} \triangleq \sum_{i=1}^{\mathbb{N}_{t}-1} S_{\mathbb{N}_{t}}^{3, \epsilon, i} .
\end{aligned}
$$

So, we have

$$
S_{\mathbb{N}_{t}}^{2, \epsilon}=\sum_{i=1}^{\mathbb{N}_{t}-1} \int_{t_{i-1}}^{t_{i}} F_{1}^{2, \epsilon}(s) d s+S_{\mathbb{N}_{t}}^{3, \epsilon}+\mathbb{R}_{\mathbb{N}_{t}}^{3, \epsilon},
$$

we can compute $S_{\mathbb{N}_{t}}^{3, \epsilon, i}$ to have

$$
S_{\mathbb{N}_{t}}^{3, \epsilon, i}=\epsilon \Delta_{t_{i-1}, t_{i}} \partial_{x} \hat{b}\left(t_{i-1}, \cdot\right) \int_{t_{i-1}}^{t_{i}} \sigma(s, s) d B_{s}+\epsilon \int_{t_{i-1}}^{t_{i}} \partial_{x} \hat{b}\left(t_{i-1}, t_{i}\right) \sigma(s, s) d B_{s}
$$

but

$$
\begin{aligned}
& \epsilon \Delta_{t_{i-1}, t_{i}} \partial_{x} \hat{b}\left(t_{i-1}, \cdot\right)=\epsilon\left(\partial_{x} \hat{b}\left(t_{i-1}, t_{i}\right)-\partial_{x} \hat{b}\left(t_{i-1}, t_{i-1}\right)\right)=\partial_{x y}^{2} \hat{b}\left(t_{i-1}, t_{i}\right) \Delta_{t_{i-1}, t_{i}} X^{\varepsilon} \\
& +\int_{0}^{1}\left(\begin{array}{c}
\partial_{x y}^{2} \hat{b}\left(X_{t_{i-1}}^{\varepsilon}, \frac{1}{\varepsilon}\left(X_{t_{i-1}}^{\varepsilon}+l \Delta_{t_{i-1}, t_{i}} X_{\cdot}^{\varepsilon}\right)\right) \\
-\partial_{x y}^{2} \hat{b}\left(t_{i-1}, t_{i}\right)
\end{array}\right) \Delta_{t_{i-1}, t_{i}} X^{\varepsilon} d l
\end{aligned}
$$


We define $U^{i} \triangleq \partial_{x y}^{2} \hat{b}\left(t_{i-1}, t_{i}\right)$,

$$
\begin{aligned}
S_{\mathbb{N}_{t}}^{4, \epsilon} \triangleq & \sum_{i=1}^{\mathbb{N}_{t}-1} \int_{t_{i-1}}^{t_{i}} U^{i} \sigma(s, s) d B_{s} \int_{t_{i-1}}^{t_{i}} \sigma(s, s) d B_{s}+\epsilon^{-1} \sum_{i=1}^{\mathbb{N}_{t}-1} \int_{t_{i-1}}^{t_{i}} U^{i} b(s, s) d s \int_{t_{i-1}}^{t_{i}} \sigma(s, s) d B_{s} \\
& +\sum_{i=1}^{\mathbb{N}_{t}-1} \int_{t_{i-1}}^{t_{i}} U^{i} c(s, s) d s \int_{t_{i-1}}^{t_{i}} \sigma(s, s) d B_{s}+\sum_{i=1}^{\mathbb{N}_{t}-1} \int_{t_{i-1}}^{t_{i}} U^{i} \gamma(s, s) d \phi_{s}^{\varepsilon} \int_{t_{i-1}}^{t_{i}} \sigma(s, s) d B_{s}, \\
\mathbb{R}_{\mathbb{N}_{t}}^{4, \epsilon} \triangleq & \sum_{i=1}^{\mathbb{N}_{t}-1} \int_{0}^{1}\left(\Delta_{i}^{l} \partial_{x y}^{2} \hat{b}\right) \Delta_{t_{i-1}, t_{i}} X^{\varepsilon} d l \int_{t_{i-1}}^{t_{i}} \sigma(s, s) d B_{s}+\epsilon \sum_{i=1}^{\mathbb{N}_{t}-1} \int_{t_{i-1}}^{t_{i}} \partial_{x} \hat{b}\left(t_{i-1}, t_{i-1}\right) \sigma(s, s) d B_{s} \\
\triangleq & \mathbb{R}_{\mathbb{N}_{t}}^{4, \epsilon, 1}+\mathbb{R}_{\mathbb{N}_{t}}^{4, \epsilon, 2},
\end{aligned}
$$

where

$$
\Delta_{i}^{l} \partial_{x y}^{2} \hat{b}=\partial_{x y}^{2} \hat{b}\left(X_{t_{i-1}}^{\varepsilon}, \frac{X_{t_{i-1}}^{\varepsilon}+l \Delta_{t_{i-1}, t_{i}} X^{\varepsilon}}{\varepsilon}\right)-\partial_{x y}^{2} \hat{b}\left(t_{i-1}, t_{i}\right)
$$

so that $S_{\mathbb{N}_{t}}^{3, \epsilon}=S_{\mathbb{N}_{t}}^{4, \epsilon}+\mathbb{R}_{\mathbb{N}_{t}}^{4, \epsilon}$. We also compute $S_{\mathbb{N}_{t}}^{4, \epsilon}$. Let us first define by $F_{3}^{3, \epsilon}(s)$ the process defined on $\left.] t_{i-1}, t_{i}\right]$ by $\operatorname{Tr}\left[\partial_{x y}^{2} \hat{b}\left(t_{i-1}, t_{i}\right) a(s, s)\right]$ and by $\left\{\mathbb{R}_{N_{t}}^{5, \epsilon, i}\right\}_{1 \leq i \leq 3}$ the last three terms in (3.34). We obtain

$$
S_{\mathbb{N}_{t}}^{4, \epsilon}=\sum_{i=1}^{\mathbb{N}_{t}-1} \int_{t_{i-1}}^{t_{i}} F_{3}^{3, \epsilon}(s) d s+\mathbb{R}_{\mathbb{N}_{t}}^{5, \epsilon},
$$

where $\mathbb{R}_{\mathbb{N}_{t}}^{5, \epsilon} \triangleq \mathbb{R}_{\mathbb{N}_{t}}^{5, \epsilon, 1}+\mathbb{R}_{\mathbb{N}_{t}}^{5, \epsilon, 2}+\mathbb{R}_{\mathbb{N}_{t}}^{5, \epsilon, 3}$

Let us now smooth out the irregularities in the construction of our coefficients. Indeed, let us write the following definition:

$$
\begin{gathered}
F(x, y) \triangleq\left(\partial_{x} \hat{b} b+\left(I+\partial_{y} \hat{b}\right) c+\operatorname{Tr}\left[\partial_{x y}^{2} \hat{b} a\right]\right)(x, y) \triangleq\left(F_{1}+F_{2}+F_{3}\right)(x, y), \\
G(x, y) \triangleq\left(\left(I+\partial_{y} \hat{b}\right) \sigma\right)(x, y), \\
\Lambda(x, y) \triangleq\left(\left(I+\partial_{y} \hat{b}\right) \gamma\right)(x, y) .
\end{gathered}
$$

Note that $F, G$, and $\Lambda$ are continuous. 
12 Locally periodic homogenization of reflected diffusion

Those operations on the coefficients of $X_{t_{*}}^{\varepsilon}$ involve the extra rests

$$
\begin{aligned}
& \mathbb{R}_{\mathbb{N}_{t}}^{6, \epsilon}=\sum_{i=1}^{\mathbb{N}_{t}-1} \int_{t_{i-1}}^{t_{i}}\left(\partial_{x} \hat{b}\left(t_{i-1}, t_{i}\right)-\partial_{x} \hat{b}(s, s)\right) b(s, s) d B_{s}, \\
& \mathbb{R}_{\mathbb{N}_{t}}^{7, \epsilon}=\int_{0}^{t_{*}}\left(\left(I+\partial_{y} \hat{b}\left(s_{*}, s\right)\right) c(s, s)-\left(I+\partial_{y} \hat{b}\right) c(s, s)\right) d s, \\
& \mathbb{R}_{N_{t}}^{8, \epsilon}=\sum_{i=1}^{\mathbb{N}_{t}-1} \int_{t_{i-1}}^{t_{i}} U^{i} \sigma(s, s) d B_{s} \int_{t_{i-1}}^{t_{i}} \sigma(s, s) d B_{s}-\int_{0}^{t_{*}} \operatorname{Tr}\left[\partial_{x y}^{2} \hat{b} a\right](s, s) d s, \\
& \mathbb{R}_{\mathbb{N}_{t}}^{9, \epsilon}=\int_{0}^{t_{*}}\left(\left(I+\partial_{y} \hat{b}\left(s_{*}, s\right)\right) \sigma(s, s)-\left(I+\partial_{y} \hat{b}\right) \sigma(s, s)\right) d B_{s}, \\
& \mathbb{R}_{\mathbb{N}_{t}, \epsilon}^{10, \epsilon}=\int_{0}^{t_{*}}\left(\left(I+\partial_{y} \hat{b}\left(s_{*}, s\right)\right) \gamma(s, s)-\left(I+\partial_{y} \hat{b}\right) \gamma(s, s)\right) d \phi_{s}^{\varepsilon} .
\end{aligned}
$$

We have thus proved the following.

Lemma 3.10. For any subdivision $t_{i}$ with constant step $\Delta t_{i}$,

$$
X_{t_{*}}^{\epsilon}=\bar{X}_{t_{*}}^{\epsilon}+\mathbb{R}_{\mathbb{N}_{t}}^{\epsilon}
$$

where

$$
\bar{X}_{t_{*}}^{\epsilon}=x+\int_{0}^{t_{*}} F(s, s) d s+\int_{0}^{t_{*}} G(s, s) d B_{s}+\int_{0}^{t_{*}} \Lambda(s, s) d \phi_{s}^{\epsilon},
$$

and the remainder $\mathbb{R}_{\mathbb{N}_{t}}^{\epsilon}$ is the sum of the residual quantities, that is,

$$
\mathbb{R}_{\mathbb{N}_{t}}^{\epsilon}=\sum_{i=1}^{10} \mathbb{R}_{\mathbb{N}_{t}}^{i, \epsilon} .
$$

We can now state the following theorem, which deals with the order of magnitude of the $\mathbb{R}_{\mathbb{N}_{t}}^{\epsilon}$.

THeOREM 3.11. With the notations above, the decomposition

$$
X_{t}^{\epsilon}=\bar{X}_{t}^{\epsilon}+\mathbb{R}_{t}^{\epsilon}, \quad t \geq 0
$$

holds, where $\bar{X}_{t}^{\epsilon}$ is the Itô process

$$
\bar{X}_{t}^{\epsilon}=x+\int_{0}^{t} F(s, s) d s+\int_{0}^{t} G(s, s) d B_{s}+\int_{0}^{t} \Lambda(s, s) d \phi_{s}^{\epsilon},
$$

and the remainder term $\mathbb{R}_{t}^{\epsilon}$ satisfies

$$
\mathbb{P}\left(\sup _{t \leq T}\left\|\mathbb{R}_{t}^{\epsilon}\right\|>\delta\right) \longrightarrow 0 \quad \forall \delta>0 .
$$


Proof. For small value of $\epsilon$, [1, Lemma 20, page 26 of the preprint] is still valid, so we use the proof of [1, Theorem 2] and we consider only the new terms, that is, $\mathbb{R}_{t}^{10, \epsilon}$ and $\mathbb{R}_{t}^{5, \epsilon, 3}$,

$$
\begin{gathered}
\mathbb{R}_{\mathbb{N}_{t}}^{10, \epsilon}=\int_{0}^{t_{*}}\left(\left(I+\partial_{y} \hat{b}\left(s_{*}, s\right)\right) \gamma(s, s)-\left(I+\partial_{y} \hat{b}\right) \gamma(s, s)\right) d \phi_{s}^{\varepsilon}, \\
\mathbb{R}_{\mathbb{N}_{t}}^{5, \epsilon, 3}=\sum_{i=1}^{\mathbb{N}_{t}-1} \int_{t_{i-1}}^{t_{i}} U^{i} \gamma(s, s) d \phi_{s}^{\varepsilon} \int_{t_{i-1}}^{t_{i}} \sigma(s, s) d B_{s},
\end{gathered}
$$

the last term clearly tends to zero in probability. We have

$$
\begin{aligned}
& \mathbb{R}_{\mathbb{N}_{t}}^{10, \epsilon}=\int_{0}^{t_{*}}\left(\partial_{y} \hat{b}\left(s_{*}, s\right)-\partial_{y} \hat{b}(s, s)\right) \gamma(s, s) d \phi_{s}^{\varepsilon}, \\
& \mathbb{E}\left(\left\|\mathbb{R}_{\mathbb{N}_{t}}^{10, \epsilon}\right\|\right) \leq c \mathbb{E}\left(\sup _{0 \leq s_{*} \leq s \leq s^{*} \leq T}\left\|X_{s}^{\varepsilon}-X_{s_{*}}^{\varepsilon}\right\|\right),
\end{aligned}
$$

hence $\left\|\mathbb{R}_{\mathbb{N}_{t}}^{10, \epsilon}\right\|$ tend to zero in probability, as $\varepsilon$ tend to zero.

In order to identify the limit points of $\mathbb{P}_{X^{\epsilon}}$, it suffices to do so for those of $\mathbb{P}_{\bar{X}^{\epsilon}}$. We use the martingale problem with reflection approach.

Remark 3.12. Following Stroock and Varadhan [12] (see also [3, pages 476-477]), the measure $\mathbb{P}_{x}^{\epsilon}$ on $\mathscr{C}([0, T], \bar{D})$ associated with $\left(L^{\epsilon}, \Gamma^{\epsilon}\right)$, where $\Gamma^{\epsilon}=\langle\gamma(\cdot, \cdot / \epsilon), \nabla\rangle$ is a solution of the submartingale problem:

(i) $\mathbb{P}_{x}^{\epsilon}(x(0)=x)=1$,

(ii) $f(x(t))-f(x(0))-\int_{0}^{t} L^{\epsilon} f(x(s)) d_{s}$ is a $\mathbb{P}_{x}^{\epsilon}$ submartingale for each $f \in \mathscr{C}^{2}(\bar{D})$ satisfying $\langle\gamma(\cdot, \cdot / \epsilon), \nabla f\rangle \geq 0$ on $\partial D$.

Under our assumptions, $\bar{X}^{\epsilon}$ (by abuse) is solution of the submartingale problem, hence $\bar{X}_{t}^{\epsilon} \in \bar{D}, t \geq 0$, and there exists a continuous, nondecreasing process $\lambda^{\epsilon}$ such that

$$
f\left(\bar{X}_{t}^{\epsilon}\right)-f\left(\bar{X}_{0}^{\epsilon}\right)-\int_{0}^{t} L^{\epsilon} f\left(\bar{X}_{s}^{\epsilon}\right) d_{s}-\int_{0}^{t}\left\langle\gamma\left(\bar{X}_{s}^{\epsilon}, \frac{\bar{X}_{s}^{\epsilon}}{\epsilon}\right), \Gamma^{\epsilon} f\left(\bar{X}_{s}^{\epsilon}\right)\right\rangle d \lambda_{s}^{\epsilon}
$$

is $\left\{\mathscr{F}_{t}^{X^{\epsilon}, \lambda^{\epsilon}}\right\}$-martingale for each $f \in C_{K}^{2}\left(\mathbb{R}^{d}\right)$ and $\int_{0}^{t} 1_{D}\left(\bar{X}_{s}^{\epsilon}\right) d \lambda_{s}^{\epsilon}=0, t \geq 0$.

Let $\varphi(x)$ be a function in $C_{K}^{\infty}\left(\mathbb{R}^{d}\right)$ and apply the Itô formula, we have for $t \geq t_{0}$,

$$
\begin{aligned}
\varphi\left(\bar{X}_{t}^{\epsilon}\right)-\varphi\left(\bar{X}_{s}^{\epsilon}\right)= & \int_{s}^{t} \partial_{x} \varphi\left(\bar{X}_{r}^{\epsilon}\right)\left[F(r, r) d r+G(r, r) d B_{r}+\Lambda(r, r) d \phi_{r}^{\epsilon}\right] \\
& +\int_{s}^{t} \operatorname{Tr} \partial_{x x}^{2} \varphi\left(\bar{X}_{r}^{\epsilon}\right) G G^{*}(r, r) d r .
\end{aligned}
$$


Now let $\Phi_{t_{0}}(\cdot), t_{0} \leq T$, be a bounded continuous functional on the Wiener space $C[0, T]$ which depends only on the past up to $t_{0}$. Define $G G^{*}=\Pi$. We have

$$
\mathbb{E}\left[\left(-\int_{t_{0}}^{t} \partial_{x} \varphi\left(\bar{X}_{r}^{\epsilon}\right)\left[\begin{array}{c}
\Delta_{t_{0}, t} \varphi\left(\bar{X}_{.}^{\epsilon}\right) \\
\left(F+\operatorname{Tr} \partial_{x x}^{2} \varphi\left(\bar{X}_{r}^{\epsilon}\right) \Pi\right)(r, r) d r \\
+\Lambda(r, r) d \phi_{r}^{\epsilon}
\end{array}\right]\right) \Phi_{t_{0}}(\cdot)\right]=0 .
$$

Let us now homogenize $F, \Pi$ and $\Lambda$ by setting (see also Section 2.2)

$$
\begin{aligned}
& \bar{C}_{0}(x)=\int_{\mathrm{T}^{d}} F(x, u) m(x, d u), \\
& \bar{A}_{0}(x)=\int_{\mathrm{T}^{d}} \Pi(x, u) m(x, d u), \\
& \gamma_{0}(x)=\int_{\mathrm{T}^{d-1}} \Lambda(x, u) \tilde{m}(x, d u) .
\end{aligned}
$$

The relation (3.50) becomes

$$
\begin{gathered}
\mathbb{E}\left[\left(-\int_{t_{0}}^{t} \partial_{x} \varphi\left(\bar{X}_{r}^{\epsilon}\right)\left[\begin{array}{c}
\Delta_{t_{0}, t} \varphi\left(\bar{X}_{.}^{\epsilon}\right) \\
\left(\bar{C}_{0}+\operatorname{Tr} \partial_{x x}^{2} \varphi\left(\bar{X}_{r}^{\epsilon}\right) \bar{A}_{0}\right)\left(X_{r}^{\epsilon}\right) d r \\
+\gamma_{0}\left(X_{r}^{\epsilon}\right) d \phi_{r}^{\epsilon}
\end{array}\right]\right) \Phi_{t_{0}}(\cdot)\right] \\
=\mathbb{E}\left[\left(\int_{t_{0}}^{t} \partial_{x} \varphi\left(\bar{X}_{r}^{\epsilon}\right)\left[\begin{array}{c}
\left(\bar{C}_{0 \#}+\operatorname{Tr} \partial_{x x}^{2} \varphi\left(\bar{X}_{r}^{\epsilon}\right) \bar{A}_{0 \#}\right)(r, r) d r \\
+\gamma_{0 \#}(r, r) d \phi_{r}^{\epsilon}
\end{array}\right]\right) \Phi_{t_{0}}(\cdot)\right],
\end{gathered}
$$

where $\bar{\zeta}_{\#}(x, y)=\zeta(x, y)-\bar{\zeta}(x)$.

Now we show the continuity of $\bar{C}_{0}, \bar{A}_{0}$, and $\gamma_{0}$.

Lemma 3.13. (i) If $x_{n} \rightarrow x_{0}$, then $m\left(x_{n}, \cdot\right) \Rightarrow m\left(x_{0}, \cdot\right)$ and the functions $\bar{C}_{0}(\cdot)$ and $\bar{A}_{0}(\cdot)$ are continuous.

(ii) If $x_{n} \rightarrow x_{0}$, then $\tilde{m}\left(x_{n}, \cdot\right) \Rightarrow \tilde{m}\left(x_{0}, \cdot\right)$ and the function $\gamma_{0}(\cdot)$ is continuous.

Proof. The weak convergence in (i) follows from [1, Lemma 6]; $\bar{C}_{0}$ and $\bar{A}_{0}$ are continuous since $F(\cdot, \cdot)$ and $\Pi(\cdot, \cdot)$ are. Let $x_{n} \rightarrow x_{0}$.

Recall that there exists a unique Markov process on $\partial D$ with generator $\Gamma^{x} H^{x}$ and By the periodicity assumption this Markov process induces a Markov process indexed by $x$ (parameter) on the torus $\mathbf{T}^{d-1}$, say $\tilde{X}^{x}$, and $\tilde{m}(x, \cdot)$ is the invariant measure of $\tilde{X}^{x}$. We have the continuity of this family of Markov processes with respect to parameter $x$, and so, if the initial point is the same, say $y$, then $\sup _{t \leq T}\left|\tilde{X}^{x_{n}}-\tilde{X}^{x_{0}}\right| \rightarrow 0$ in probability by [8, Theorem 7.1] (or see Gīhman and Skorohod [7, pages 54-55] for details). Now,

$$
\forall \varphi \in \mathscr{b}\left(T^{d-1}\right), 0 \leq t \leq T, \quad \int_{\mathrm{T}^{d-1}} \varphi(y) \tilde{m}\left(x_{n}, d y\right)=\int_{\mathrm{T}^{d-1}} \mathbb{E}^{y}\left[\varphi\left(\tilde{X}_{t}^{x_{n}}\right)\right] \tilde{m}\left(x_{n}, d y\right)
$$


hence

$$
\int_{\mathrm{T}^{d-1}} \varphi(y) \tilde{m}\left(x_{n}, d y\right)=\int_{\mathrm{T}^{d-1}} \mathbb{E}^{y}\left[\varphi\left(\tilde{X}_{t}^{x_{0}}\right)\right] \tilde{m}\left(x_{n}, d y\right)+\int_{\mathrm{T}^{d-1}} \mathbb{E}^{y}\left[\varphi\left(\tilde{X}_{t}^{x_{n}}\right)-\varphi\left(\tilde{X}_{t}^{x_{0}}\right)\right] \tilde{m}\left(x_{n}, d y\right) .
$$

The last term tends to zero, as $n \rightarrow \infty$, consequently, we obtain the identity

$$
\int_{\mathrm{T}^{d-1}} \varphi(y) \tilde{m}_{0}(d y)=\int_{\mathrm{T}^{d-1}} \mathbb{E}^{y}\left[\varphi\left(\tilde{X}_{t}^{x_{n}}\right)\right] \tilde{m}_{0}(d y) \quad \forall \varphi \in \mathscr{b}\left(T^{d-1}\right), 0 \leq t \leq T .
$$

Hence $\tilde{m}_{0}=\tilde{m}\left(x_{0}, \cdot\right)$, and the whole sequence $\left\{\tilde{m}\left(x_{n}, \cdot\right)\right\}_{n>0}$ converges to $\tilde{m}\left(x_{0}, \cdot\right)$.

The function $\Lambda(x, y)$ is continuous so we deduce that

$$
\lim _{n \rightarrow \infty} \gamma_{0}\left(x_{n}\right)=\lim _{n \rightarrow \infty} \int_{\mathrm{T}^{d-1}} \Lambda\left(x_{n}, u\right) \tilde{m}\left(x_{n}, d u\right)=\int_{\mathbf{T}^{d-1}} \Lambda\left(x_{0}, u\right) \tilde{m}\left(x_{0}, d u\right)=\gamma_{0}\left(x_{0}\right) .
$$

Now we need a locally period theorem to deal with the expected value on the righthand side in (3.52). This can be done by the two following lemmas.

LEMma 3.14. Let $h(x, y)$ be a continuous bounded function on $\mathbb{R}^{d} \times \mathbf{T}^{d}$ such that for all $x \in \mathbb{R}^{d}$,

$$
\int_{\mathrm{T}^{d}} h(x, y) m(x, d y)=0,
$$

$h(x, \cdot) \in \mathbf{W}^{1, p}\left(\mathbf{T}^{d}\right)$ for some $p>d$, and moreover there exists $c(p)<\infty$, such that

$$
\|h(x, \cdot)\|_{\mathbf{W}^{1, p}\left(\mathbf{T}^{d}\right)} \leq c(p) .
$$

Suppose moreover that $\varphi \in \mathscr{C}_{K}^{\infty}\left(\mathbb{R}^{d}\right)$, then

$$
H^{\epsilon}(t)=\int_{0}^{t} \varphi\left(\bar{X}_{s}^{\epsilon}\right) h\left(X_{s}^{\varepsilon}, \frac{X_{s}^{\varepsilon}}{\varepsilon}\right) d s
$$

converge to zero in $L^{1}(\Omega)$ for any $0<t \leq T$.

Proof. See the careful proof of [1, Theorem 3] and Lemma 3.5.

Lemma 3.15. Let $v(x, y)$ be a continuous bounded function on $\mathbb{R}^{d} \times \mathbf{T}^{d-1}$ such that for all $x \in \mathbb{R}^{d}$,

$$
\int_{\mathrm{T}^{d-1}} v(x, y) \tilde{m}(x, d y)=0 .
$$

Suppose moreover that $\varphi \in \mathscr{C}_{K}^{\infty}\left(\mathbb{R}^{d}\right)$, and for $y=(0, \tilde{y}) \in \mathbf{T}^{d}, \tilde{y} \in \mathbf{T}^{d-1}$, (but here abuse by $y \in \mathbf{T}^{d-1}$ )

$$
H^{\epsilon}(t)=\int_{0}^{t} \varphi\left(\bar{X}_{s}^{\epsilon}\right) v\left(X_{s}^{\varepsilon}, \frac{X_{s}^{\varepsilon}}{\varepsilon}\right) d \phi_{r}^{\epsilon}
$$

converge to zero in $L^{1}(\Omega)$ for any $0<t \leq T$. 
Proof. Since $\varphi \in \mathscr{C}_{K}^{\infty}\left(\mathbb{R}^{d}\right)$ and $\bar{X}^{\epsilon} \Rightarrow X$, it suffices to prove that $\int_{0}^{t} v\left(X_{s}^{\varepsilon}, X_{s}^{\varepsilon} / \varepsilon\right) d \phi_{r}^{\epsilon}$ converge to zero in $L^{1}(\Omega)$ for any $0<t \leq T$, and to treat the case of $v$ continuous with compact support in $\mathbb{R}^{d} \times \mathbf{T}^{d-1}$. Since such a function can be uniformly approximated by a function of the form

$$
\sum_{1}^{n} h_{i}(x) g_{i}(y)
$$

it is sufficient to establish the result for $v$ of the form

$$
v(x, y)=h(x) g(y)
$$

with $h$ and $g$ continuous and bounded. One can take $h>0$ (since the result is still for $-v$ ).

Since all integrands are bounded, we have $\left|H^{\epsilon}(t)\right| \leq K \phi_{t}^{\epsilon}$ so $\sup _{\varepsilon}\left|H^{\epsilon}(t)\right|^{2}<\infty$, it is sufficient to establish that $\sup _{0 \leq t \leq T}\left|H^{\epsilon}(t)\right| \rightarrow 0$ in probability as $\varepsilon \rightarrow 0$.

We have $\int_{0}^{t} v\left(X_{s}^{\varepsilon}, X_{s}^{\varepsilon} / \varepsilon\right) d \phi_{r}^{\epsilon}=\int_{0}^{t} h\left(X_{s}^{\varepsilon}\right) g\left(X_{s}^{\varepsilon} / \varepsilon\right) d \phi_{r}^{\epsilon}$, let $d \varphi_{r}^{\epsilon}=h\left(X_{s}^{\varepsilon}\right) d \phi_{r}^{\epsilon}$ and $d \varphi_{r}=h\left(X_{s}\right) d \phi_{r}$ so that $\int_{0}^{t} h\left(X_{s}^{\varepsilon}\right) g\left(X_{s}^{\varepsilon} / \varepsilon\right) d \phi_{r}^{\epsilon}=\int_{0}^{t} g\left(X_{s}^{\varepsilon} / \varepsilon\right) d \varphi_{r}^{\epsilon}$.

Since $\left\{Z_{t}^{\varepsilon} \triangle=h\left(X_{t}^{\varepsilon}\right): 0 \leq t \leq T\right\}$ converges in the same sense as $\left\{X_{t}^{\varepsilon}\right\}$, now $h(x)>0$ so $\varphi^{\epsilon}, \varphi . \in V_{+}^{c}$ follows clearly from the fact (see the proof of [9, Lemma 3.3]) that the mapping

$$
(z, \phi) \longrightarrow \int_{0}^{t} z d \phi
$$

is continuous from $D([0, T]) \times V_{+}^{c}$ into $C([0, T])$, where we equip $D([0, T])$ with the $S$ topology, $V_{+}^{c}$ and $C([0, T])$ with the sup-norm topology. Here and below $V_{+}$denotes the set of càdlàg increasing functions from $[0, T]$ into $\mathbb{R}$ and $V_{+}^{c}$ the set of continuous increasing functions from $[0, T]$ into $\mathbb{R}$. Let

$$
\begin{gathered}
\bar{g}=\left(\inf _{y \in \mathbf{T}^{d-1}} g(y)\right)^{-}, \\
\tilde{g}=\int_{T^{d-1}}(g(y)+\bar{g}) \tilde{m}(x, d y) .
\end{gathered}
$$

It suffices to prove that

$$
\int_{0}^{t}\left(g\left(\frac{X_{s}^{\varepsilon}}{\varepsilon}\right)+\bar{g}\right) d \varphi_{s}^{\varepsilon} \stackrel{\mathscr{L}}{\longrightarrow} \tilde{g} \int_{0}^{t} d \varphi_{s}
$$

as $\varepsilon \rightarrow 0$. But $\left(\int_{0}^{t}\left(g\left(X_{s}^{\varepsilon} / \varepsilon\right)+\bar{g}\right) d \varphi_{s}^{\varepsilon} ; 0 \leq t \leq T\right)_{\mathcal{\varepsilon} \backslash 0}$ is a collection of increasing functions which converge in law uniformly in $t$ to the continuous function $\left(\tilde{g} \varphi_{t} ; 0 \leq t \leq T\right)$, see Tanaka [13, Lemma 6.4].

Theorem 3.16. There is only one limit point of the family $X^{\epsilon}$ as $\epsilon \rightarrow 0$, namely $X^{0}$, the diffusion with reflection solution of

$$
X_{t}^{0}=x+\int_{0}^{t} \bar{A}_{0}^{1 / 2}\left(X_{s}^{0}\right) d W_{s}+\int_{0}^{t} \bar{C}_{0}\left(X_{s}^{0}\right) d s+\int_{0}^{t} \gamma_{0}\left(X_{s}^{0}\right) d \lambda_{s}
$$

where $\bar{C}_{0}(x), \bar{A}_{0}(x)$, and $\gamma_{0}(x)$ are defined in (3.51) (see also (3.26), (3.38)). 
Proof. Since $\bar{C}_{0}(x), \bar{A}_{0}(x)$, and $\gamma_{0}(x)$ are continuous, it suffices, in order to be able to apply uniqueness theorem to a constrained martingale problem to show as in the martingale uniqueness problem, that the matrix $\bar{A}_{0}(x)$ is positive definite for each $x$. This last point is shown in the proof of [1, Corollary 2].

\section{Acknowledgments}

The first author was supported by an AUF postdoctoral Grant and would like to express his deep gratitude to Ouknine and UCAM Mathematics Department for their friendly hospitality.

\section{References}

[1] A. Benchérif-Madani and É. Pardoux, Homogenization of a diffusion with locally periodic coefficients, Lecture Notes in Mathematics, 2004, pp. 397-409.

[2] __Locally periodic homogenization, Asymptotic Analysis 39 (2004), no. 3-4, 263-279.

[3] A. Bensoussan, J.-L. Lions, and G. C. Papanicolaou, Asymptotic Analysis for Periodic Structures, Studies in Mathematics and Its Applications, vol. 5, North-Holland, Amsterdam, 1978.

[4] P. Billingsley, Convergence of Probability Measures, John Wiley \& Sons, New York, 1968.

[5] M. I. FreĬdlin, The Dirichlet problem for an equation with periodic coefficients depending on a small parameter, Teorija Verojatnosteĭ i ee Primenenija 9 (1964), 133-139.

[6] Markov Processes and Differential Equations: Asymptotic Problems, Lectures in Mathematics ETH Zürich, Birkhäuser, Basel, 1996.

[7] ̆. İ. Gīhman and A. V. Skorohod, Stochastic Differential Equations, Springer, New York, 1972.

[8] A. G. Malliaris, Stochastic Methods in Economics and Finance, Advanced Textbooks in Economics, vol. 17, North-Holland, Amsterdam, 1982, with a foreword and contributions by W. A. Brock, eight reprint 1999.

[9] Y. Ouknine and É. Pardoux, Homogenization of PDEs with non linear boundary condition, Ascola Conference, 2004, pp. 293-307.

[10] É. Pardoux, Homogenization of linear and semilinear second order parabolic PDEs with periodic coefficients: a probabilistic approach, Journal of Functional Analysis 167 (1999), no. 2, 498-520.

[11] L. Słomiński, Euler's approximations of solutions of SDEs with reflecting boundary, Stochastic Processes and Their Applications 94 (2001), no. 2, 317-337.

[12] D. W. Stroock and S. R. S. Varadhan, Diffusion processes with boundary conditions, Communications on Pure and Applied Mathematics 24 (1971), 147-225.

[13] H. Tanaka, Homogenization of diffusion processes with boundary conditions, Stochastic Analysis and Applications, Advanced Probability in Related Topics, vol. 7, Dekker, New York, 1984, pp. 411-437.

Aboubakary Diakhaby: UFR SAT, Université Gaston Berger, BP 234, Saint-Louis, Senegal

E-mail addresses: diakhaby@ugb.sn; diakhaby81@yahoo.fr

Youssef Ouknine: Department of Mathematics, Faculty of Sciences Semlalia, BP 2390,

Marrakesh, Morocco

E-mail addresses: ouknine@ucam.ac.ma; ouknine@yahoo.com 


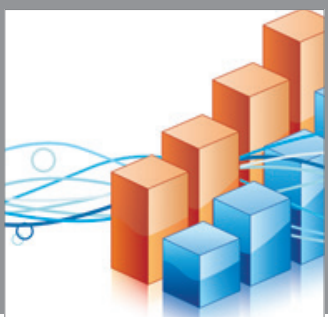

Advances in

Operations Research

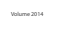

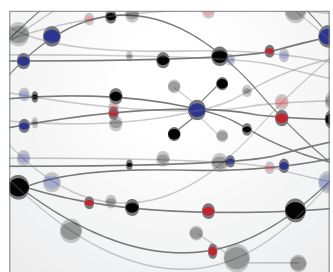

\section{The Scientific} World Journal
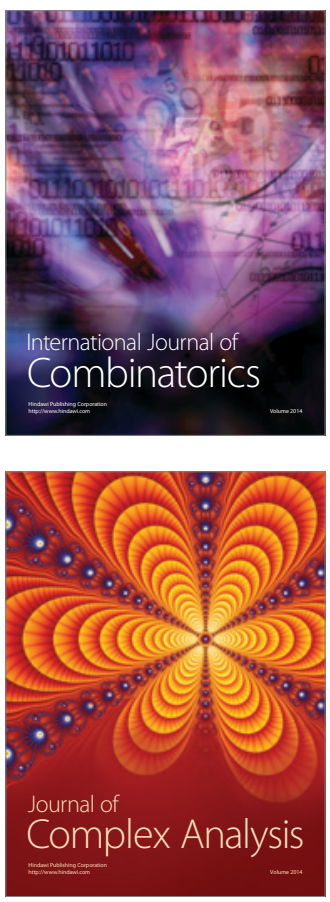

International Journal of

Mathematics and

Mathematical

Sciences
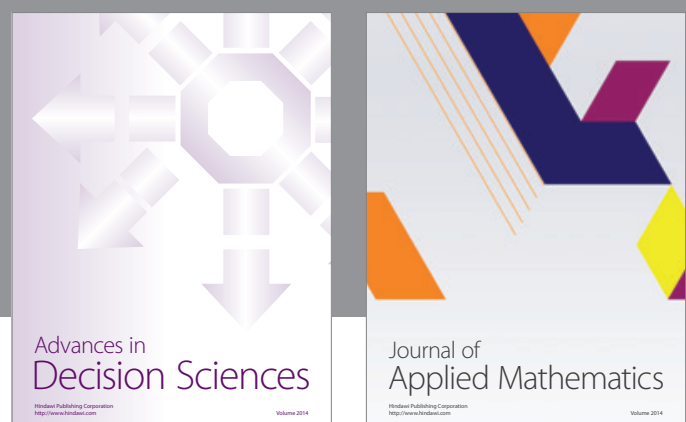

Journal of

Applied Mathematics
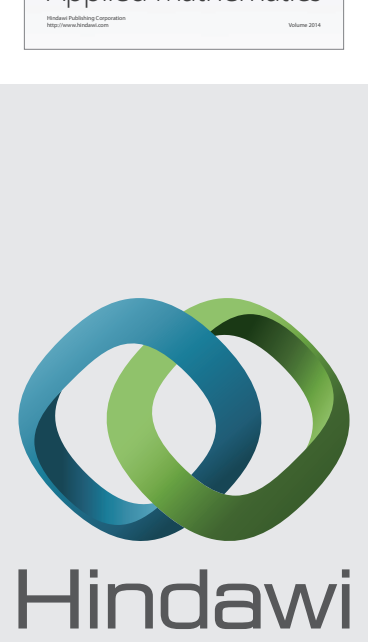

Submit your manuscripts at http://www.hindawi.com
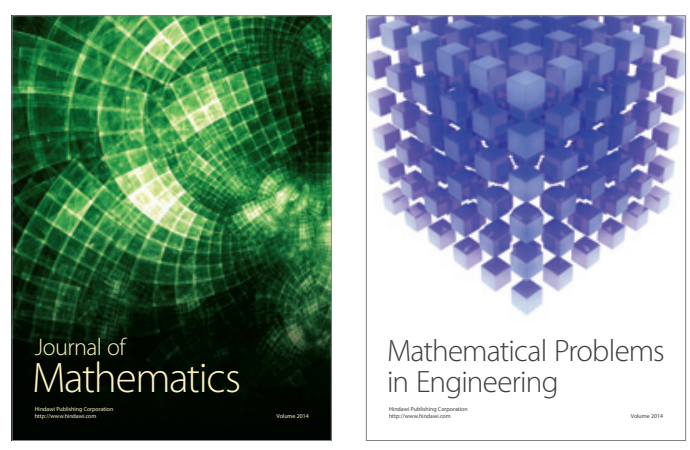

Mathematical Problems in Engineering
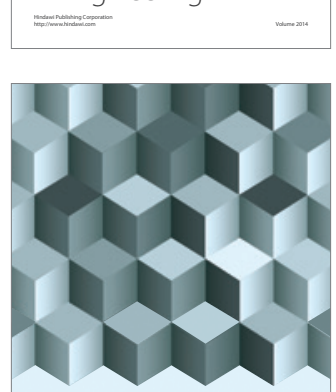

Journal of

Function Spaces
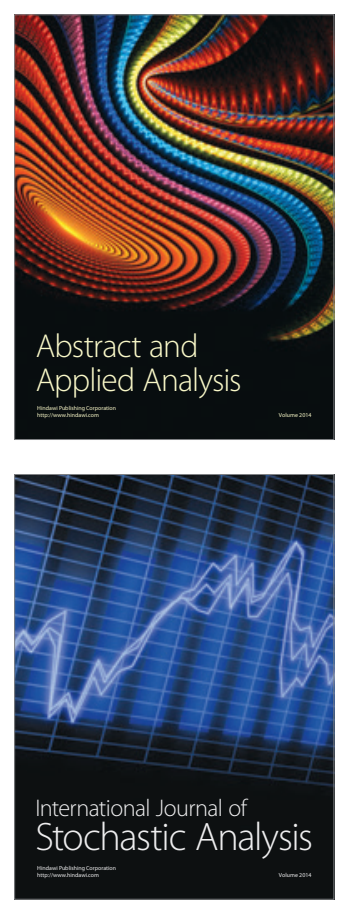

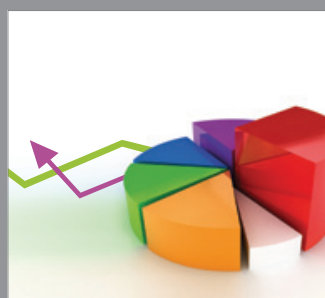

ournal of

Probability and Statistics

Promensencen
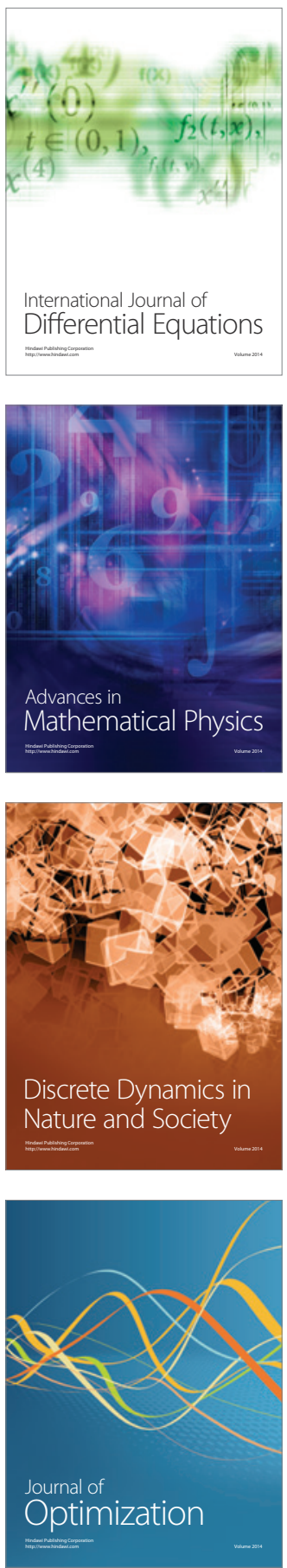